\title{
IMAGE PROCESSING FOR MATERIALS CHARACTERIZATION: ISSUES, CHALLENGES AND OPPORTUNITIES
}

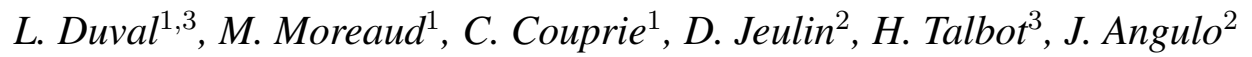 \\ ${ }^{1}$ IFP Energies nouvelles \\ ${ }^{2}$ MINES ParisTech, PSL Research \\ ${ }^{3}$ Université Paris-Est
LIGM, ESIEE Paris
France \\ Technology, Comp. Science and Applied Mathematics Dept. \\ France \\ Centre de Morphologie Mathématique \\ France \\ France
}

\begin{abstract}
This introductory paper aims at summarizing some problems and state-of-the-art techniques encountered in image processing for material analysis and design. Developing generic methods for this purpose is a complex task given the variability of the different image acquisition modalities (optical, scanning or transmission electron microscopy; surface analysis instrumentation, electron tomography, micro-tomography...), and material composition (porous, fibrous, granular, hard materials, membranes, surfaces and interfaces...). This paper presents an overview of techniques that have been and are currently developed to address this diversity of problems, such as segmentation, texture analysis, multiscale and directional features extraction, stochastic models and rendering, among others. Finally, it provides references to enter the issues, challenges and opportunities in materials characterization.
\end{abstract}

Index Terms - Image Processing, Image-based Analysis, Materials science, Stochastic modeling, Surface Science, Texture Analysis, Work-flow

\section{INTRODUCTION}

\subsection{Context}

Periods in mankind's history are often named after specific materials [1]. The classic ages are termed after Stone, Bronze or Iron. More recently, industrial breakthroughs remain intimately related to particular materials. Steam and electricity, as drivers of the first and second industrial revolutions, were intimately associated with steel [2]. Silicon partly shaped the information and communication area. The initial choice of a material is nowadays crucial for the development of innovative technologies. First, large investments are required to make it pervasive. Also, tensions relating to the availability of natural resources exist, as well as pressing needs in fast technological cycles.

Materials technology is evolving from materials discovered in nature by chance to predicted and designed materials [3, 4], that repair themselves, adapt to their environment, capture and store energy or information, help enable new devices and sensors, etc. Materials are now designed from scratch with initial blueprints, starting from atoms and molecules. Materials [1] impacted include ceramics, composites [5], carbon, glass, metals and alloys, polymers, etc. This evolution, at the confluence of science, technology, and engineering [6], is driven by the synergy of materials science and physics, mechanics, chemistry, biology and engineering. J. W. Gibbs (1839-1903), who was awarded the first American doctorate in engineering, may personify this synergy. He is better known in the signal/image processing community for the characterization of oscillatory behaviors in Fourier series. He also demonstrated crucial relationships between properties of materials in different phases and their thermodynamics at the atomic structure level [7].

Materials pervade many areas: energy production, transportation, construction, textiles and packaging, biology, to name a few. Materials engineering, or materials science, stemmed from metallurgy to a fast-growing, specific field of science. Although not its main application, image processing is progressively taking part in this challenge [8]. Moreover, with the increasing precision of instrumentation, down to the direct observation of atomic processes at the sub-Ångström scale, evolving over picoseconds [9], the data flow is likely to require a tremendous effort from the image processing community, involving various topics from compression [10] to restoration [11], to the standardization of processing work-flows on different image channels or modalities [12]. This possibility is backed in [13]:

It is my strong belief that all branches of materials science, including "materials" such as snow, ice and biological tissue, form a very important, perhaps the most important, field of application of the methods of stochastic-geometry based image analysis and spatial statistics, which aims to describe irregular structures statistically by numerical or functional summary characteristics.

Indeed, the possibility of designing, analyzing and modeling materials from images (or generally two- or threedimensional modalities) reveals important contributions to this field. The appearance of materials changes significantly with imaging techniques, depending on the scale of analysis, imaging settings, physical properties and preparation of materials. Understanding these aspects turns out to be crucial for material analysis and modeling. In particular, we face challenges regarding the characterization of the physical assembly process of materials, the formation process of images, of imaging techniques interacting with materials (geometry, transmission, illumination, reflection, scattering).

Answering these questions is important to separate the material appearance from its intrinsic morphology and properties. 
Complemented with invited researchers of complementary expertise, from image feature extraction to image simulation, this paper aims to report on issues, challenges and emerging trends in material analysis and modeling that are likely to pose challenges to image processing, through the diversity of types of materials, acquisition techniques and applications. Indeed, in many cases, the traditional, human, vision-based interpretation of materials image is misleading, as illustrated in the serendipitous face and crescent moon appearance in Fig. 1. Fully taking into account physical properties and laws is at the heart of successful image analysis in material science.

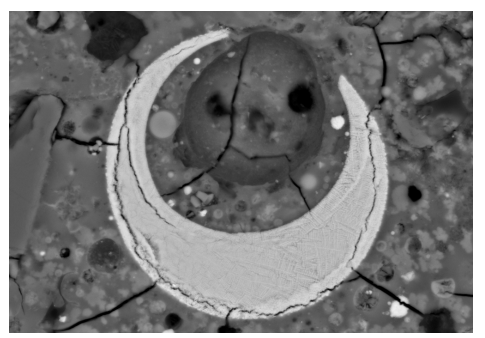

Fig. 1. Scanning electron microscopy: Polymer-charged concrete (C)Florent Moreau, IFPEN).

\subsection{Scope}

This introductory paper aims at showing some relevant problems in material characterization that can be addressed with classical or advanced methods from signal and image processing. It provides a large overview of some of the issues that may be addressed in this application domain, such as dealing with different modalities and applications such as clean energy and information storage; chemistry and catalysts; geology; forensics; bio-inspired materials and biomedical [14]). For illustrating and to widen the points of view, some of these challenges can be undertaken by employing active sub-fields of image processing, e.g. restoration and inverse problems [15]; segmentation $[16,17,18]$; texture analysis $[19,20]$; multiscale and directional features extraction methods [21]; color and multispectral processing [12]; stochastic models [22, 23].

The paper is organized as follows: Section 2 provides introductory examples, to illustrate a part of the variety of materials data. It also details goals assigned to image processing tasks. Section 3 details traditional as well as complementary approaches, with selected references. Some conclusions on the main challenges are given in Section 4.

\section{AIMS AND MOTIVATIONS}

\subsection{Introductory examples}

Figures 2-7 represent a sample of images acquired and problems for material analysis. Fig. 2 illustrates the cracks (black), partially filled pores (rounded dark gray shapes with white spots) and zeolite inclusions (scattered or aggregated white dots) in a neutral substrate (alumin). Its characteristics relate to the efficiency of a spherical catalyst used in refining for the conversion of hydrocarbons into chemical products. Such images raise different problems at different scales of observations, from isolated objects to scattered phases, requiring different segmentation tools. Fig. 3 results from optical microscopy. The thickness of the crusts may be measured by electron probe microanalysis. It directly correlates with catalyst activity. Resorting to image processing techniques [24] to automate the measurement of crust thickness avoids invasive probe techniques, and hastens the conception of new catalysts [24]. In Fig. 4, one can use local orientation estimates for 2D and $3 \mathrm{D}$ texture segmentation. In Fig. 5, the goal is to infer bounds on the effective properties of materials by dealing with three-dimensional, computational microstructure simulations. It combines a multiscale approach with homogenization techniques [25]. The same goal is pursued in Fig. 6, here at the nanometric scale. Fig. 7 illustrates a mixture of a crystalline and an amorphous material. The periodicity of the first one, and the lack of long-term regularity of the second one, drives the use of local Fourier attributes [26].

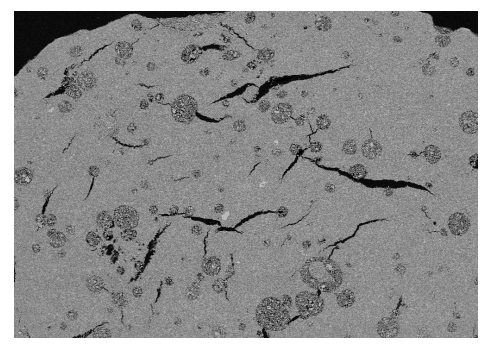

Fig. 2. Scanning electron microscopy: catalyst section (C)IFPEN).

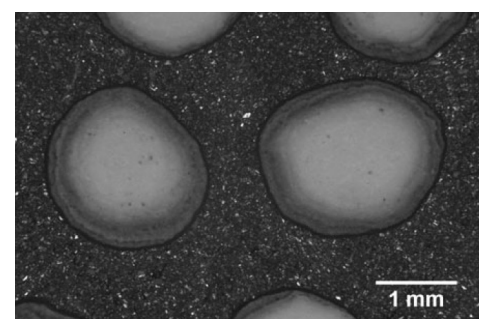

Fig. 3. Catalysts with metallic palladium crust (C)IFPEN).

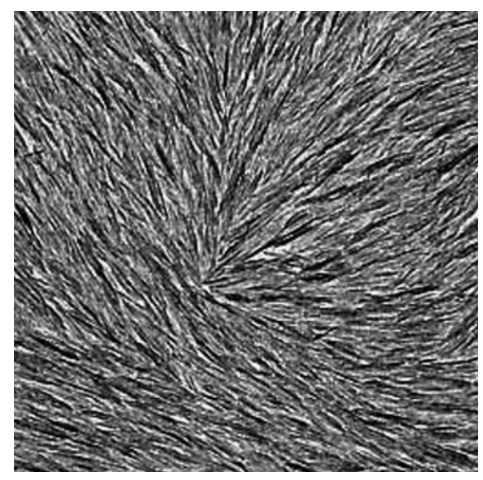

Fig. 4. Cellulose whisker observed in transmission electron microscopy. (C)J.-L. Putaux, Cermav, CNRS). 


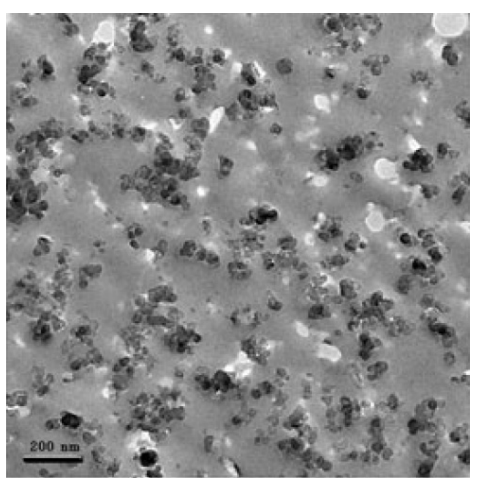

Fig. 5. Filled rubber's microstructures (@)Michelin).

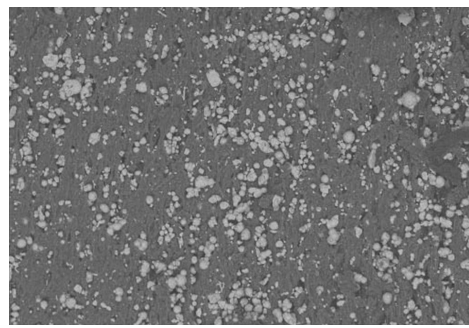

Fig. 6. Composite material with elastomer matrix (C)EADS).

\subsection{Ultimate goals}

Image processing should ultimately allow:

- a refined understanding of structured two-dimensional or volumetric data to improve the extraction of truly useful material characteristics,

- an efficient analysis of larger data volume through processing automation.

Several goals are together of interest in material design:

- to improve existing materials with specific properties: global structure (morphology, porosity), microstructure (grains, fibers, pores [8]), mechanical or thermal resistance, electrical or magnetic traits, diffusion...

- to enhance a production process (cost, resources, flow) maintaining determined physical properties,

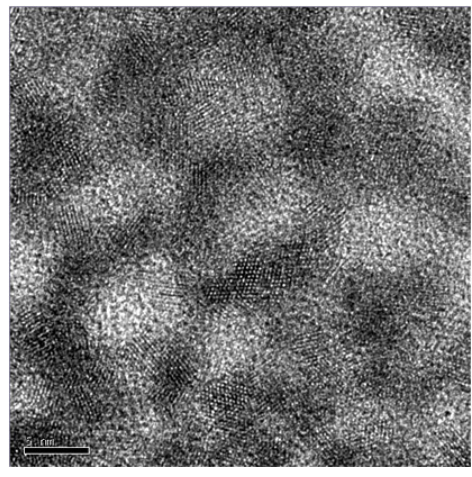

Fig. 7. Atomic structure of a ceria nanoparticle (C)Rhodia).
- to monitor quality, with material comparison, defect and rare events detection and characterization, stress or corrosion resistance.

These aspects may be directly linked to the microstructure. In this case, images provide precise and quantitative information and allow the computation of (often geometric) criteria. Alternatively, these aspects are related to both the microstructure and specific properties of the material components. Image processing then serves as a pre-processing permitting the estimation of physical properties and parameters through homogenization. Such methods require as an input a description of the microstructure as fine as possible, as well as intrinsic properties of material components. Image processing enables better understanding of properties or behaviors of materials, reducing the resort to physical testings, the latter being generally more involved, slower or more expensive, and destructive. In certain cases, specific information can be obtained only though image processing methods, for instance for 3D connexity at the nanometer scale in electronic tomography. These outputs should permit to adapt or rectify manufacturing settings toward optimal physical properties. In this context, image-processingtuned stochastic models play a crucial role.

The ultimate goals of image processing for materials characterization concentrate to form a strong link in a chained experimental process striving to predict and improve materials, in a fast and more accurate manner.

\section{IMAGE PROCESSING: ISSUES AND CHALLENGES}

\subsection{Classical approaches and techniques}

One may define a relatively standard work-flow for image processing in materials science. It includes the following steps:

Image acquisition or reconstruction Depending on the resolution of interest, different equipments may be used, for instance optical microscopes $(\mathrm{mm}-\mu \mathrm{m})$, scanning electron microscopes or SEM ( $\mu \mathrm{m},[27])$ and transmission electron microscopes or TEM (nm-Å, [28]) for 2D acquisitions. Turning to 3D acquisitions [29], one observes a frequent use of X-ray tomography $(\mathrm{mm}-\mu \mathrm{m})$ and electronic tomography $(\mathrm{nm})$ based on TEM projections [30]. Some techniques deal with the reconstruction of surface topography [20].

Enhancement and filtering This step (Fig. 2, with bilateral filtering) relates to image improvement from a noise level or homogeneity point-of-view. One is primarily interested in noise filtering techniques permitting only minimal modifications in the structures of interest (e.g. bilateral filters [31], morphological filtering [32, 33]), or drift or background correction. Techniques for artifact corrections, specific to the acquisition process, include for instance ring artifact suppression (X-ray tomography), sample impregnation or encasement in colorless resin (causing effects akin to cast shadows), mechanical or ionic surface polishing. 
Segmentation This step (Fig. 3, with watershed), encompassing dimensionality reduction, classification and model fitting, simplifies rich images into a few meaningful intensities or components. It aims at the recovery of the border of the objects of interest. When the colorintensity level is directly related to object properties (topography, like in secondary electron SEM, or chemical structure, like in back-scattered electron SEM [34]), global histogram segmentation methods (inter-class variance maximization, entropy, Expectation-Maximization) are complemented by criterion-based segmentation (object length, surface, tortuosity). This combination permits the elimination of non conform or non interesting elements, sometimes mere residuals of a previous processing sequence. For images whose intensity is less directly related to properties, extraction of local morphology or contours may be performed using global optimization approaches such as geodesic active contours and regions [35], maximum flows approaches [36] and/or mathematical morphology operations [37, 38] such as top-hat filtering or watershed transformations $[39,40,41]$.

Analysis Image structures (Fig. 6) can be described without an explicit segmentation of the objects of interest using multiscale image representations based on morphological decompositions [42] or wavelet-based multiscale representations [43, 44]. Once simplified, objects of interest may be characterized by numbers, shapes and spatial distribution among others. Stereology [45], stochastic modeling [46] (spatial moments, such as the covariance, and spatial distributions) and the use of distance functions (inter-object, geodesic length, tortuosity) provide the main sources of algorithms.

Multi-modality It involves image regularization and segmentation of multi-modal/multichannel/multiscale images acquired from the same samples. In order to deal with this kind of data, some pre-processing steps such as registration [47], denoising [48] and image fusion are required. In addition to extract relevant information from complex data sets integrating various acquisition techniques, it is useful to use dimensionally reduction techniques, from classical PCA to modern nonlinear manifold learning $[49,50]$.

Microstructure stochastic modeling This step (Fig. 5, with 3D modeling from 2D images) may use input characteristic data either from segmentation or analysis. It consists in estimating a stochastic, geometric model (Boolean random functions, reaction-diffusion or dilution models, dead leaves tessellations), representing the microstructure of the observed material. It serves the purposes of inferring the potential 3D structure from 2D cuts, or of obtaining structure variations through model parameter modifications, hence of "numerical material by design". Finally, this step may even replace the segmentation step, when individual objects can not, or need not, be individualized [51].

\subsection{Advanced and complementary approaches}

Aside from the main topics exposed above, complementary approaches are listed below.

Segmentation and analysis are sometimes merged. Loosely termed "texture approaches", those methods aim at extracting image characteristics without resorting to individual or excessively precise object separation. Local orientations (Fig. 4 and Fig. 7), meaningful alignments in microstructures, morphological metrics are extracted, with the help of 2D/3D models. Machine learning techniques are also emerging, typically to automate supervised/unsupervised classification problems [52].

Meaningful characteristics may be enriched by combining or merging different acquisition techniques. Acquisitions may consider different scales of observation (multiscale fusion approaches), or different modalities (multi-modal fusion approaches), or both. Such techniques, very common in medical or satellite imaging, are increasingly being used in materials design, combining for instance topographic and physico-chemical information (via secondary electron and back-scattered SEM or Energy-Dispersive Spectroscopy).

With 3D or 3D+time imaging [9], an important effort is dedicated to processing performance, especially algorithm acceleration. Materials science processing work-flows are increasingly carried out, with reduced cost, with CPU/GPU optimization [53]. For instance, tomographic reconstruction is typically now faster than acquisition. Similar performance increases are being pursued with filtering or segmentation techniques, toward integrated high-throughput materials science and engineering.

\section{CONCLUSIONS}

Among the challenges proposed before, and the ones recently exposed in [9], we retain the attention on the following four:

- multi-step: to produce a complete pipe-line or work-flow of image analyses and simulations, from sample acquisition to the estimation of physical properties;

- data-flow: to manage raw and simulated data and acceleration of the aforementioned work-flows from high throughput screening of materials with ever increasing amounts of numerical information;

- multi-modality (or synergy): to employ as much as possible the potentialities to retrieve information on materials from different acquisition sources, with novel fusion/combination techniques improving characterization or providing access to novel properties;

- reverse engineering: to help in computational material design [54], by orienting the synthesis or manufacturing process of novel materials, desired or predicted [55] (e.g. graphene, silicene).

A successful application of more standard image processing thus requires a better integration of known techniques, as well as the deployment of image analysis not used in materials science yet, with the new challenges raised by its variety of data and physical models. 


\section{REFERENCES}

[1] R. E. Hummel, Understanding Materials Science: History, Properties, Applications, Springer, 2nd edition, 2004.

[2] J. A. Mathews, “The steel age-1876 to 1926," Ind. Eng. Chem., vol. 18, no. 9, pp. 913-914, 1926

[3] P. Ball, Made to Measure: New Materials for the 21st Century, Princeton University Press, 1999.

[4] Y. Bréchet, "Materials science: from materials discovered by chance to made-tomeasure materials," Collège de France, 2013, Chair of Technological Innovation.

[5] J. P. Davim and C. A. Charitidis, Eds., Nanocomposites: materials, manufacturing, and engineering, Advanced Composites. Walter De Gruyter, Berlin Boston, 2013.

[6] Y. Liu and X. Zhang, "Metamaterials: a new frontier of science and technology," Chem. Soc. Rev., vol. 40, no. 5, pp. 2494, 2011.

[7] J. W. Gibbs, "On the equilibrium of heterogeneous substances," Trans. Connect. Acad. Arts Sci., vol. III, pp. 108-248 and 343-524, 1875 to 1878.

[8] L. Wojnar, Image analysis. Applications in materials engineering, CRC Press, 1999.

[9] I. M. Robertson, C. A. Schuh, J. S. Vetrano, N. D. Browning, D. P. Field, D. J. Jensen, M. K. Miller, I. Baker, D. C. Dunand, R. Dunin-Borkowski, B. Kabius, T. Kelly, S. Lozano-Perez, A. Misra, G. S. Rohrer, A. D. Rollett, M. L. Taheri, G. B. Thompson, M. Uchic, X.-L. Wang, and G. Was, "Towards an integrated materials characterization toolbox," J. Mater. Res., vol. 26, no. 11, pp. 1341-1383, Jun 2011.

[10] D. Salomon and G. Motta, Handbook of Data Compression, Springer, 2009.

[11] B. K. Gunturk and X. Li, Eds., Image Restoration: Fundamentals and Advances, Digital Imaging and Computer Vision. CRC Press, 2012.

[12] J. Chanussot, C. Collet, and K. Chehdi, Eds., Multivariate Image Processing, ISTE Ltd and John Wiley \& Sons Inc., 2009.

[13] J. Ohser and K. Schladitz, 3D Images of Materials Structures: Processing and Analysis, Wiley-VCH, 2009

[14] A. Depeursinge, A. Foncubierta-Rodriguez, D. Van De Ville, and H. Müller, "Threedimensional solid texture analysis in biomedical imaging: Review and opportunities," Med. Image Anal., vol. 18, no. 1, pp. 176-196, 2014.

[15] H. H. Bauschke, R. S. Burachik, P. L. Combettes, V. Elser, D. R. Luke, and H. Wolkowicz, Eds., Fixed-Point Algorithms for Inverse Problems in Science and Engineering, Springer, 2011.

[16] M. L. Comer and E. J. Delp, "The EM/MPM algorithm for segmentation of textured images: analysis and further experimental results," IEEE Trans. Image Process., vol. 9, no. 10, pp. 1731-1744, 2000.

[17] C. Chaux, A. Jezierska, J.-C. Pesquet, and H. Talbot, "A spatial regularization approach for vector quantization," J. Math. Imaging Vis., vol. 41, no. 1-2, pp. 23-38, 2011.

[18] C. Park, J. Z. Huang, J. X. Ji, and Y. Ding, "Segmentation, inference and classification of partially overlapping nanoparticles," IEEE Trans. Patt. Anal. Mach. Int., vol. 35 , no. 3, pp. 669-681, 2013.

[19] M. Petrou and P. G. Sevilla, Image processing: dealing with texture, John Wiley \& Sons, Ltd, 2006.

[20] R. Leach, Ed., Characterisation of Areal Surface Texture, Springer, 2013.

[21] S. T. Ali, J.-P. Antoine, and J.-P. Gazeau, Multidimensional Wavelets and Generalizations, pp. 411-456, Theoretical and Mathematical Physics. Springer-Verlag, Aug. 2014.

[22] S. Torquato, Random Heterogeneous Materials: Microstructure and Macroscopic Properties, Springer, 2002.

[23] D. Jeulin and M. Ostoja-Starzewski, Eds., Mechanics of Random and Multiscale Microstructures, Springer, 2001.

[24] L. Sorbier, A.-S. Gay, A. Fécant, M. Moreaud, and N. Brodusch, "Measurement of Palladium crust thickness on catalysts by optical microscopy and image analysis," Microsc. Microanal., vol. 19, pp. 293-299, 42013.

[25] A. Jean, D. Jeulin, S. Forest, S. Cantournet, and F. N'Guyen, "A multiscale microstructure model of carbon black distribution in rubber," J. Microsc., vol. 241, no. 3, pp. 243-260, Mar. 2011.

[26] M. Moreaud, D. Jeulin, A. Thorel, and J. Y. Chane-Ching, "A quantitative morphological analysis of nanostructured ceria-silica composite catalysts," J. Microsc., vol. 232, no. 2, pp. 293-305, Nov. 2008.

[27] L. Reimer, Scanning Electron Microscopy: Physics of Image Formation and Microanalysis, Springer Series in Optical Sciences. Springer, 2013.

[28] L. Reimer and H. Kohl, Transmission Electron Microscopy: Physics of Image Formation, vol. 36 of Springer Series in Optical Sciences, Springer, 5th edition, 2008.
[29] S. F. Li and R. M. Suter, "Adaptive reconstruction method for three-dimensional orientation imaging," J. Appl. Crystallogr, vol. 46, no. 2, pp. 512-524, 2013.

[30] V.-D. Tran, M. Moreaud, É. Thiébaut, L. Denis, and J.-M. Becker, "Inverse problem approach for the alignment of electron tomographic series," Oil Gas Sci. Tech. 2013.

[31] C. Tomasi and R. Manduchi, "Bilateral filtering for gray and color images," in Proc. IEEE Int. Conf. Comput. Vis., Jan. 1998, pp. 839-846.

[32] J. Angulo, "Morphological bilateral filtering," SIAM J. Imaging Sci., vol. 6, no. 3 , pp. 1790-1822, 2013.

[33] J. Angulo and S. Velasco-Forero, "Stochastic morphological filtering and BellmanMaslov chains," in Mathematical Morphology and Its Applications to Signal and Image Processing, C. L. L. Hendriks, G. Borgefors, and R. Strand, Eds., vol. 7883 of Lect. Notes Comput. Sci., pp. 171-182. Springer Berlin Heidelberg, 2013.

[34] A. J. Schwartz, M. Kumar, D. P. Field, and B. L. Adams, Eds., Electron backscatter diffraction in materials science, Springer, 2009.

[35] N. Paragios and R. Deriche, "Geodesic active regions and level set methods for supervised texture segmentation," Int. J. Comp. Vis., vol. 46, no. 3, pp. 223-247, 2002.

[36] B. Appleton and H. Talbot, "Globally minimal surfaces by continuous maximal flows," IEEE Trans. Patt. Anal. Mach. Int., vol. 28, no. 1, pp. 106-118, 2006.

[37] J. Goutsias, L. M. Vincent, and D. S. Bloomberg, Eds., Mathematical morphology and its applications to image and signal processing, Kluwer academic, 2002.

[38] L. Najman and H. Talbot, Eds., Mathematical Morphology, Wiley, 2010.

[39] S. Beucher and F. Meyer, "The morphological approach to segmentation: the watershed transformation.," Opt. Eng., vol. 34, pp. 433-481, 1993.

[40] Y. Tarabalka, J. Chanussot, and J. A. Benediktsson, "Segmentation and classification of hyperspectral images using watershed transformation," Pattern Recogn., vol. 43 , no. 7, pp. 2367-2379, Jul. 2010.

[41] C. Couprie, L. Grady, L. Najman, and H. Talbot, "Power watershed: A unifying graph-based optimization framework," IEEE Trans. Patt. Anal. Mach. Int., vol. 33, no. 7, pp. 1384-1399, Jul. 2011.

[42] S. Velasco-Forero and J. Angulo, "Classification of hyperspectral images by tensor modeling and additive morphological decomposition," Pattern Recogn., vol. 46, no. 2, pp. 566-577, Feb. 2013.

[43] B. Vidakovic, Statistical Modeling by Wavelets, Wiley-Interscience, 1999.

[44] L. Jacques, L. Duval, C. Chaux, and G. Peyré, "A panorama on multiscale geometric representations, intertwining spatial, directional and frequency selectivity," Signal Process., vol. 91, no. 12, pp. 2699-2730, Dec. 2011.

[45] A. Baddeley and E. B. Vedel Jensen, Stereology for statisticians, Number 103 in Monographs on Statistics and Applied Probability. Chapman \& Hall/CRC, 2005.

[46] G. Gaiselmann, R. Thiedmann, I. Manke, W. Lehnert, and V. Schmidt, "Stochastic 3D modeling of fiber-based materials," Comput. Mater. Sci., vol. 59, pp. 75-86, 2012.

[47] B. Pan, K. Qian, H. Xie, and A. Asundi, "Two-dimensional digital image correlation for in-plane displacement and strain measurement: a review," Meas. Sci. Tech., vol 20, no. 6, pp. 062001, Jun. 2009.

[48] C. Chaux, L. Duval, A. Benazza-Benyahia, and J.-C. Pesquet, "A nonlinear Stein based estimator for multichannel image denoising," IEEE Trans. Signal Process. vol. 56, no. 8, pp. 3855-3870, Aug. 2008.

[49] H. Edelsbrunner and J. L. Harer, Computational Topology: An Introduction, American Mathematical Society, 2009, Departments of Computer Science and Mathematics, Duke University.

[50] A. Cord, F. Bach, and D. Jeulin, "Texture classification by statistical learning from morphological image processing: application to metallic surfaces," J. Microsc., vol. 239 , no. 2, pp. 159-166, 2010.

[51] M. Moreaud, D. Jeulin, V. Morard, and R. Revel, "TEM image analysis and modelling: application to boehmite nanoparticles," J. Microsc., vol. 245, no. 2, pp. 186-199, 2012.

[52] J. Michopoulos, "Mechatronically automated characterization of material constitutive response," in Proceedings of the 6th World Congress on Computational Mechanics (WCCM-VI), Beijing China, September 5-10 2004, pp. 486-491, Tsinghua University Press and Springer.

[53] R. Couturier, Ed., Designing Scientific Applications on GPUs, CRC Press, 2013

[54] S. Curtarolo, G. L. W. Hart, M. Buongiorno Nardelli, N. Mingo, S. Sanvito, and O. Levy, "The high-throughput highway to computational materials design," Nat. Mater, vol. 12, pp. 191-201, Mar. 2013.

[55] A. K. Geim and K. S. Novoselov, "The rise of graphene," Nat. Mater., vol. 6, no. 3, pp. 183-191, Mar. 2007. 